\title{
Necesidad de colaboración en investigación entre cardiólogos y médicos de urgencias
}

\section{Need for research collaboration between cardiologists and emergency service doctors}

\section{Ò. Miró ${ }^{1}$}

\section{RESUMEN}

El tiempo es un factor clave en el abordaje de las enfermedades y los síndromes cardiológicos agudos. La organización actual de los sistemas sanitarios hace que los primeros minutos de asistencia no se lleven a cabo en salas de cardiología ni dependan de cardiólogos, sino que se producen en el entorno prehospitalario por sistemas de emergencias médicas (SEM) y en servicios de urgencias hospitalarios (SUH), y en primera instancia esta atención es proporcionada por médicos de urgencias. Por tanto, para obtener el mejor resultado posible para el paciente, es necesario que exista una colaboración extensa y bidireccional entre cardiólogos y médicos de urgencias. El presente artículo analiza estas necesidades e intenta identificar áreas de mejora en dos de los procesos cardiológicos agudos más frecuentes: el síndrome coronario agudo y la insuficiencia cardiaca aguda.

Palabras clave. Urgencias. Cardiología. Multidisciplinar. Síndrome coronario agudo. Insuficiencia cardiaca aguda.

\begin{abstract}
Time is a key factor when tackling acute coronary diseases and syndromes. The current organization of health services means that the first minutes of care are not carried out in cardiology rooms and do not depend on cardiologists. Instead they take place in the pre-hospital setting of emergency medical services (EMS) and in hospital accident and emergency services (A\&E) and in the first phase this attention is provided by emergency doctors. Therefore, to obtain the best possible result for the patient it is necessary that there should be extensive two-way collaboration between cardiologists and emergency service doctors. This article analyzes these needs and tries to identify areas of improvement in two of the most frequent acute coronary processes: acute coronary syndrome and acute cardiac insufficiency.
\end{abstract}

Key words. Emergency services. Cardiology. Multidisciplinary. Acute coronary syndrome. Acute cardiac insufficiency.
1. Área de Urgencias, Hospital Clínic de Barcelona. Grupo de investigación "Urgencias: Procesos y Patologías”, IDIBAPS. Barcelona.

Recepción: 13 de enero de 2014

Aceptación provisional: 14 de febrero de 2014

Aceptación definitiva: 10 de marzo de 2014

\author{
Correspondencia: \\ Òscar Miró \\ Área de Urgencias \\ Hospital Clinic \\ Villarroel, 170 \\ 08036 Barcelona \\ E-mail: omiro@clinic.cat
}




\section{INTRODUCCIÓN}

En el argot deportivo, se dice que hay que jugar bien desde el primer minuto si se quiere ganar un partido. Esta máxima deportiva universal bien puede aplicarse al ejercicio de la medicina en general y al de la cardiología en particular. Está claro que en el abordaje de las enfermedades y los síndromes cardiológicos agudos, el factor tiempo es clave para alcanzar el mejor resultado posible. Y dada la organización que los sistemas sanitarios han alcanzado durante las últimas décadas, este primer minuto no siempre se juega en las salas de cardiología, sino en el domicilio del paciente, la vía pública y las unidades de soporte vital avanzado (USVA) de manos de la atención prehospitalaria que facilitan los sistemas de emergencias médicas (SEM) ${ }^{1-3}$, $y$, por otra parte, en los boxes de primera asistencia de unos a menudo saturados servicios de urgencias hospitalarios $(\mathrm{SUH})^{4-6}$. Es comprensible, pues, que en este escenario sus actores, cardiólogos y médicos de urgencias, hayan decidido estrechar lazos, desarrollando alianzas y definiendo estrategias comunes con el fin último de ganar el partido. Este artículo repasa sucintamente algunas iniciativas llevadas a cabo en España durante los últimos años en estos terrenos de juego, donde los SEM y los SUH empiezan a tomar parte activa en la investigación $^{7-10}$. Esta investigación es fundamental para mejorar esos primeros minutos, entre otras circunstancias, en dos situaciones cardiológicas altamente relevantes por su incidencia y morbimortalidad asociadas: el síndrome coronario agudo (SCA) y la insuficiencia cardiaca aguda (ICA).

\section{EL SÍNDROME CORONARIO AGUDO: UN EJEMPLO DE NECESIDAD DE COLABORACIÓN}

\section{El dolor torácico en los servicios de urgencias}

El SCA siempre ha sido un reto diagnóstico en el paciente que consulta por dolor torácico. El dolor torácico no traumático supone alrededor del 5\% de las consultas en los SUH y, de éstos, un 10\% presenta un infarto de miocardio, con o sin elevación del segmento ST (IAMCEST e IAMSEST, respectivamente) y hasta un $15 \%$ adicional presenta algún otro tipo de $\mathrm{SCA}^{11}$. Esto supone que aproximadamente un paciente por cada 100 que acuden al SUH tendrá un SCA. Las dificultades para la identificación de estos pacientes empiezan por la propia historia clínica, la cual no siempre es fácil. La responsabilidad inicial de realizarla correctamente recae en primera instancia en el médico de urgencias por lo que, para él, ahondar en el conocimiento de los aspectos vinculados a la semiología del dolor torácico en general y del SCA en particular es fundamental. Esto es especialmente relevante en el caso de las mujeres, en las que se ha comprobado de forma repetida y también en España que presentan, con respecto a los hombres, una sintomatología más atípica y una peor identificación de los síntomas como correspondientes a un posible $\mathrm{SCA}^{12-14}$. Ello ha conducido a una demora en el aviso a los SEM y a una llegada más tardía a los SUH, incluso para aquellas pacientes que presentan un SCA con elevación del segmento ST (SCACEST) ${ }^{14}$. Sin embargo, en un análisis realizado por Riesgo A y col en 97 SUH españoles incluidos en el registro MUSICA, los autores comprobaron que estas diferencias en la percepción del dolor y en el retraso de solicitud de asistencia médica no se tradujeron en una asistencia más deficiente. Así, la tendencia a menos ingresos en unidades de coronariasintensivos y menos cateterismos urgentes encontrada en ellas desapareció tras el ajuste por las diferencias en las características basales con respecto a los hombres ${ }^{13}$. Con todo, se hace necesario ahondar en todos los aspectos que permitan acortar los tiempos empleados, especialmente en las mujeres, para proporcionar la primera asistencia a los pacientes con dolor torácico.

\section{El abordaje y el tratamiento del síndrome coronario agudo en los servicios de urgencias}

Durante estos primeros minutos de la asistencia médica al SCA, también existen áreas de mejora en cuanto a la administra- 
ción de tratamientos. Así, en el citado trabajo de Riesgo y col se vio cómo un $10 \%$ de los pacientes con un IAMSEST no recibió aspirina, un $15 \%$ no se trató con heparina y en casi el $50 \%$ no se había administrado la dosis de carga de clopidogrel durante su atención urgente por parte de los SEM y/o los $\mathrm{SUH}^{14}$. Se ha visto además que estas oportunidades de mejora varían en función del tamaño del hospital en el que es atendido el paciente, como también ha demostrado el registro MUSICA ${ }^{15}$. Efectivamente, a pesar que las características basales del paciente y las del episodio de SCA sin elevación del segmento ST (SCASEST) no muestran diferencias clínicamente relevantes entre hospitales, el tratamiento en urgencias se inicia significativamente más tarde en los hospitales de tercer nivel que en los de primer y segundo nivel, y aquéllos, a pesar que administran aspirina en urgencias con mayor frecuencia, la carga de clopidogrel y el tratamiento anticoagulante también son proporcionados con menor frecuencia en sus SUH. El análisis de los factores relacionados con estas desigualdades y la puesta en marcha de los mecanismos correctores que minimicen el alejamiento de los SUH de los estándares de tratamiento suponen dos áreas de investigación e intervención prioritaria.

Más allá del SCACEST, que se identifica rápidamente en base a los hallazgos del ECG, la detección precoz del resto de pacientes con SCA sigue representando un reto en los SUH. A día de hoy, se desconoce en números reales el alcance de los pacientes no diagnosticados de SCA y dados de alta de los SUH españoles con una valoración incompleta o insuficiente. El advenimiento de las troponinas ultrasensibles ha supuesto un gran avance en este sentido por su elevada sensibilidad y precocidad a la vez que ha permitido acortar los tiempos asistenciales y de inicio de tratamiento en los $\mathrm{SUH}^{16}$. Debe tenerse en cuenta, sin embargo, la necesidad de realizar un buen diagnóstico diferencial basado en la historia clínica, dado que este biomarcador, aunque muy sensible, no es específico de SCA y su interpretación errónea en ocasiones puede conllevar retrasos diagnósticos y terapéuticos en los propios $\mathrm{SUH}^{16,17}$. Estudios de éste y otros biomarcadores de daño miocárdico potencialmente utilizables en el futuro para el diagnóstico del dolor torácico deberán llevarse a cabo necesariamente en estos escenarios: los SEM y los SUH. Un ejemplo reciente lo constituye el estudio COPED-MIRRO llevado a cabo en 28 SUH españoles, en el que ha podido demostrarse cómo la determinación de copeptina en el momento que se extrae sangre para la primera determinación de troponina no permite descartar con seguridad el SCA en los pacientes con un ECG no diagnóstico y en los que esta primera troponina ha sido normal y, por tanto, no evita la necesidad de que los pacientes permanezcan en el SUH para una segunda determinación de troponina $^{18}$. Además, en una extensión de este mismo proyecto, el estudio COPED-PAO demostró que tampoco la copeptina añade información pronóstica adicional a la que ofrece la troponina per $s e^{19}$. Estos resultados permiten ver cómo una misma pregunta, planteada desde la perspectiva de las necesidades que tienen los $\mathrm{SUH}$, no necesariamente conduce a la misma respuesta que cuando se plantea desde otra óptica ${ }^{20}$. En este mismo sentido de intentar mejorar el rendimiento y el tiempo diagnósticos en los SUH, se está evaluando actualmente la tomografía computarizada cardiaca ${ }^{21}$. Si bien su aplicación no es generalizada, ni en cuanto a pacientes (se circunscribiría a los pacientes de alto riesgo de SCA sin patología coronaria previa conocida) ni en cuanto a centros (solo está disponible en hospitales de tercer nivel), la definición de su papel final dentro del entramado del diagnóstico diferencial del dolor torácico en los SUH está por definir y el diseño de protocolos de aplicación, acordados entre cardiólogos y médicos de urgencias, es un campo de investigación conjunta en el que debiera profundizarse ${ }^{21}$.

Por otra parte, existen situaciones particulares en las que la atención ofrecida por los SEM y los SUH cobra mayor relevancia si cabe a la hora de definir los cuadros clínicos, delimitar los pacientes de riesgo y protocolizar actitudes diagnóstico-terapéuticas. Tal es el caso, por ejemplo, del dolor 
torácico asociado al consumo de cocaína. Estos pacientes, nada infrecuentes en los SUH españoles, en muchas ocasiones son dados de alta desde los propios SUH sin ingresar y sin consultar con el cardiólogo, aun cuando su riesgo de reconsulta sea elevado $^{22}$. Y además, el consumo de cocaína en esta situación no es todavía bien apreciado por los profesionales que participan en su atención. Así, cuando se estudiaron pacientes mayores de 18 años atendidos por dolor torácico en dos SUH españoles, se detectaron metabolitos de cocaína en orina en el $6,4 \%$, y ninguno de estos pacientes fue ingresado en el hospital ${ }^{23}$. Adicionalmente, la investigación fisiopatológica de las particularidades asociadas a este tipo de SCA requiere de la participación de los médicos de urgencias ${ }^{24,25}$, máxime si se tiene en cuenta que la toxicología clínica es un área fuertemente asociada a la practica de la medicina de urgencias y emergencias ${ }^{26}$.

\section{La atención prehospitalaria al síndrome coronario agudo}

Con todo, los mayores retos y oportunidades de colaboración y mejora en el tratamiento en los primeros instantes asistenciales del SCA se presentan en la asistencia prehospitalaria que proporcionan los SEM. Por las peculiaridades que el modelo asistencial comporta en sí mismo, las capacidades investigadoras de los SEM han sido tradicionalmente poco explotadas ${ }^{8}$. Un ejemplo puede ser el mejor conocimiento de lo que acontece durante los primeros minutos de una parada cardíaca y las posibilidades de intervenciones de mejora. Los registros estructurados, con carácter general en un territorio determinado y siguiendo el modelo Utstein, deben ayudar a progresar en este sentido, y de hecho ya se están llevando a cabo ${ }^{27,28}$. Otros aspectos asistenciales y de tratamiento en el entorno extrahospitalario también pueden mejorarse, como recientemente demostraba un trabajo realizado por Hernández García y col en el que ponían de manifiesto una concordancia diagnóstica del $75 \%$ entre tres USVA y los hospitales de referencia donde transferían a los pacientes con do- lor torácico, si bien en el caso del SCACEST la concordancia era del $100 \%{ }^{29}$. Además, cuando existía la sospecha de SCA, los autores encontraron que el uso de aspirina (74\%) y nitratos (84\%) no era universal, y el de morfina (28\%), clopidogrel (24\%) y heparina (17\%) cabía considerarlos bajos. Esto cobra una máxima relevancia si se tiene en cuenta que cuando el tratamiento es proporcionado de forma adecuada, precoz y completa, éste asocia importantes mejoras en el pronóstico de los pacientes con SCASEST $^{30}$. Sin embargo, debe reconocerse que la puesta en marcha del código infarto en algunas grandes ciudades españolas ha supuesto una mejora en el tratamiento de los pacientes a la vez que una distribución de recursos y oportunidades más igualitaria ${ }^{1,31}$. Datos procedentes del área metropolitana sur de Barcelona, por ejemplo, muestran que la puesta en marcha de este código de activación desde los SEM ha permitido reducir en más de un $25 \%$ el tiempo que transcurre desde el primer contacto médico del paciente con un SCA hasta la apertura de la arteria coronaria afectada ${ }^{31}$. Durante los próximos años deberá seguir este análisis de los resultados alcanzados con esta estrategia desde la visión conjunta de cardiólogos y médicos de urgencias para seguir mejorando, a la vez que para plantearse y responder a nuevas cuestiones en este ámbito prehospitalario de actuación de los SEM.

\section{La insuficiencia cardiaca aguda: relevancia del abordaje en urgencias}

Por su lado, la ICA es un síndrome en cuya atención participan un gran número de profesionales y de especialidades médicas. Sin embargo, el pronóstico de estos pacientes no ha cambiado sustancialmente durante las últimas décadas a pesar de que se han ensayado diversos tratamientos, y por ello su manejo terapéutico sigue basándose esencialmente en la administración de oxígeno y diuréticos en una gran proporción de pacientes. La definición del espectro de este síndrome casi siempre ha resultado sesgado por el hecho de que las series han seleccionado pacientes contro- 
lados en unidades especializadas o pacientes ingresados (bien globalmente, bien en servicios específicos como salas de cardiología, de medicina interna o unidades coronarias o de cuidados intensivos) pero rara vez han tenido una perspectiva global. Con ello se detraen del cuadro clínico aquellos pacientes valorados en los SUH y dados de alta sin necesidad de ingreso, ya sea directamente o ya sea a través de sus salas de observación. Esto sucede entre una tercera y una cuarta parte del total de pacientes diagnosticados de ICA en los $\mathrm{SUH}^{32}$. Estos pacientes dados de alta desde los SUH tienen sin duda unas características diferenciales, marcadas por su mayor edad, su mayor dependencia funcional y por tratarse de formas más leves de descompensación de su insuficiencia cardiaca crónica. En ellos, además, identificar los factores pronóstico resulta más difícil. Por ello, no debieran olvidarse cuando se pretende abordar el mejor conocimiento de este síndrome. En este sentido, los SUH constituyen un marco único para llevar a cabo estos estudios con pretensión de alcanzar a todo el universo de pacientes, pues prácticamente la totalidad de pacientes con un episodio de ICA consultarán en él.

Se hace patente, pues, la necesidad que tienen de trabajar conjuntamente desde el primer minuto, entre otros especialistas, médicos de urgencias y cardiólogos. Sin entrar a profundizar en las posibilidades que esta colaboración ofrece, a continuación se ejemplifican una serie de estudios realizados desde los SUH españoles. Por ejemplo, se ha visto que los pacientes que presentan una infección respiratoria como causa de descompensación de la ICA tienen un mejor pronóstico que aquéllos en los que no se identifica un factor precipitante ${ }^{33}$. Por otro lado, el estudio PICASU-I ha permitido comprobar la capacidad pronóstica evolutiva que tiene la determinación de péptidos natriuréticos (BNP) con carácter urgente a los pacientes que consultan con ICA en el los SUH españoles en cuanto a predecir mortalidad y reconsultas preco$\mathrm{ces}^{34}$, a la vez que el estudio PICASU-II ha demostrado que dicha determinación con carácter urgente no tiene ninguna repercu- sión a la hora de mejorar el pronóstico de estos pacientes ${ }^{35}$. En efecto, los SUH españoles que disponen de esta posibilidad no alcanzan mejores resultados que los que no la tienen, lo cual posiblemente hace patente la necesidad de definir actitudes de tratamiento y disposición de los pacientes (alta o ingreso, y ubicación de este ingreso cuando es preciso) de forma diferenciada en función de los valores obtenidos de BNP. Asimismo, el uso de nuevos tratamientos o de nuevas indicaciones de fármacos ya presentes en el mercado (como es el caso de tolvaptán ${ }^{36}$ ) precisa en algunos casos de la participación de los SUH por la necesidad de precocidad en su utilización, ya que por lo general, el grueso de pacientes con ICA mejoran a las pocas horas de la llegada al Servicio de Urgencias su sintomatología con el mero uso de oxígeno y diuréticos.

$Y$ esta necesidad de colaboración se hace más evidente si cabe en el caso de los ensayos clínicos. Durante los últimos años, algunos SUH españoles han realizado ensayos clínicos independientes ${ }^{37,38}$ y actualmente se están incorporando a los ensayos clínicos promovidos por la industria en colaboración con los cardiólogos y otros especialistas hospitalarios, como internistas o intensivistas. Este protagonismo creciente deriva, por una parte, de la evolución madurativa que han experimentado en cuanto a capacidad investigadora los médicos de urgencias y los $\mathrm{SUH}^{39}, \mathrm{y}$, por otra, de la necesidad de inclusión lo más precoz posible de estos pacientes a dichos protocolos tras su llegada al servicio de Urgencias. Como se ha discutido anteriormente, la mayor parte del beneficio clínico de los tratamientos de la ICA se produce en las primeras horas, y si el paciente no es incluido en el ensayo hasta su llegada a una sala de hospitalización, convencional o de cuidados intensivos, se pierde gran parte del potencial beneficio del fármaco en cuestión, amén de un número no despreciable de pacientes que potencialmente se podrían incluir en el protocolo.

Muchos de estos resultados se han alcanzado gracias al desarrollo del registro EAHFE, en el cual participan de forma activa 29 SUH españoles ${ }^{32-35}$. Este registro, iniciado 
en 2007 y que ha contado hasta la fecha con tres fases sucesivas de inclusión de pacientes, ha recogido hasta la actualidad 5.845 pacientes consecutivos con ICA, y constituye una buena base en la que plantear hipótesis y contrastarlas. En febrero de 2014 se inició una cuarta fase de recogida de datos en la que esperamos plantear, de la mano de cardiólogos y otros especialistas que intervengan en su manejo, nuevas preguntas. Somos conscientes, los que participamos activamente en dicho registro, que necesitamos de su ayuda para poder obtener el máximo rendimiento a un esfuerzo realizado desde y por los SUH. Es difícil recoger registros de base cuasi universal (posiblemente el registro EAHFE lo sea por lo que respecta a la ICA), por lo que no seria comprensible no poner a disposición de todos los actores intervinientes en la atención de los pacientes con ICA todas las capacidades de los SUH. $\mathrm{Al}$ igual que sucedía con el SCA, en la ICA existen muchas preguntas que plantear (y muchas hipótesis que demostrar) que se juegan en los minutos iniciales de la atención al paciente.

En conclusión, los médicos de urgencias siempre hemos confiando en la altísima capacidad de las salas de hospitalización y de los especialistas que en ellas trabajan para conseguir unos magníficos resultados en los pacientes que atendemos. Sin embargo, unos y otros cada vez somos más conscientes que el partido comienza en el primer minuto, y que este primer minuto lo juegan los SEM y los SUH. Por ello, solo intensificando las complicidades entre todos los jugadores podremos ganar siempre el partido... o al menos, obtener el mejor resultado posible.

\section{BIBLIOGRAFÍA}

1. Jiménez Fàbrega X, Espila Etxeberria JL, Gallardo Mena J. Códigos de activación: pasado, presente y futuro en España. Emergencias 2011; 23: 311-318.

2. Pacheco A, Burusco S, Senosiáin MV. Prevalencia de procesos y patologías atendidos por los servicios de emergencia médica extrahospitalaria en España. An Sist Sanit Navar 2010; 3: 37-46.
3. Ablitas JM, González Lorente P, GoienetXe A, Istúriz A, Biurrun J, CASADAmón L et al. Nuevo sistema de geolocalización en Navarra para disminuir los tiempos de respuesta en aviso urgente en zonas de montaña y de gran dispersión. An Sist Sanit Navar 2013; 36: 47-55.

4. SÁnchez M, Miró O, Coll-Vinent B, Bragulat E, Espinosa G, Gómez-Angelats E et al. Emergency department overcrowding: quantification of associated factors. Med Clin (Barc) 2003; 121: 161-172.

5. Julián-Jiménez A, González del Castillo J, Martínez Ortíz de ZÁrate M, Candel González FJ, PIÑERA SAlmerón P, Moya Mir M. Características y cambios epidemiológicos de los pacientes con neumonía adquirida en la comunidad en los servicios de urgencias hospitalarios. An Sist Sanit Navar 2013; 36: 387-396.

6. Sánchez M, Santiago I. Áreas organizativas especíFICAS Y CIRCUITOS PREFERENTES PARA PATOLOGÍAS PREVALENTES EN URGENCIAS. An Sist Sanit Navar 2010; 33: 89-96.

7. Piñera Salmerón P. La investigación en red en Medicina de Urgencias y Emergencias. Emergencias 2012; 24: 234-237.

8. Rosell-Ortiz F, Mateos Rodríguez AA, Miró O. La investigación en medicina de urgencias y emergencias prehospitalaria. Emergencias 2012; 24: 3-4.

9. Jiménez Murillo L, Montero Pérez FJ. Complejidad de la asistencia médica urgente en la España del siglo XXI. An Sist Sanit Navar 2010; 33 (Supl.1): 7-11.

10. Toranzo T, Aramburu F. Los profesionales de urgencias y emergencias: perfil actual y posibilidades de desarrollo. An Sist Sanit Navar 2010; 33 (Supl.1): 13-18.

11. Bragulat E, López B, Miró O, Coll-Vinent B, Jiménez S, Aparicio MJ et al. Performance assessment of an emergency department chest pain unit. Rev Esp Cardiol. 2007; 60: 276-284.

12. Carbajosa Dalmau J, Llorens Soriano P, Diéguez Zaragoza S, Carratalá Perales JM, Díaz Dámaso J, Martínez Beloqu E et al. Influencia del sexo del paciente en el manejo del síndrome coronario agudo con elevación del ST en los servicios de urgencias. Emergencias 2011; 23: 87-92.

13. Riesgo A, Miró O, López-de-SÁ E, SÁnchez M. Comparison of the management of non-ST segment elevation myocardial infarction during emergency care according to sex of the patient. Rev Esp Cardiol 2011; 64: 1060-1064.

14. Bolívar Muñoz J, Martínez Cassinello R, Mateo Rodríguez I, Torres Ruiz JM, Pascual Martínez N, Rosell Ortiz F et al. Actuación de los pacientes ante un síndrome coronario agudo: diferencias desde una perspectiva de género. Emergencias 2013; 25: 23-30. 
15. Sánchez M, Bauset Navarro JL, Cuervo Pinto R, Carbajosa J, Oria MC, Povar Marco J et al. Atención médica al síndrome coronario agudo sin elevación del ST en los servicios de urgencias según el tamaño del hospital receptor. Contribuciones del registro MUSICA. Emergencias 2011; 23: 447-454.

16. Ordóñez Llanos J, Santaló Bell M. Diagnóstico precoz de la lesión miocárdica: ¿Qué nos depararán los marcadores bioquímicos en el futuro? Emergencias 2011; 23: 149-151.

17. Estella A. Dolor torácico no coronario con biomarcadores cardiacos elevados: presentación de 15 casos compatibles con miocarditis aguda. Emergencias 2011; 23: 375-377.

18. Llorens P, Sánchez M, Herrero P, Martín-SÁnchez FJ, PIÑERA P, Miró O et al. The utility of copeptin in the emergency department for non-STelevation myocardial infarction rapid rule out: COPED-MIRRO study. Eur J Emerg Med 2014; (en prensa).

19. Sánchez M, Llorens P, Herrero P, Martín-Sanchez FJ, PIÑERA P, MiRó O ET AL. The utility of copeptin in the emergency department as a predictor of adverse outcomes in non-ST-elevation acute coronary syndrome: the COPED-PAO study. Emerg Med J 2014; 31: 286-291.

20. Reichlin T, Hochholzer W, Stelzig C et al. Incremental value of copeptin for rapid rule out of acute myocardial infarction. J Am Coll Cardiol 2009; 54: 60-68.

21. Miró O, Tomás Ortiz-Pérez J, León R, LóPez Seoane B, Perea R, De Caralt TM et al. Estudio piloto de la utilidad de la tomografía computarizada cardiaca para descartar síndrome coronario agudo en urgencias. Emergencias 2010; 22: 101-108.

22. Galicia M, Nogué S, Casañas X, Iglesias ML, Puiguriguer J, Supervía A et al. Multicenter assessment of the revisit risk for a further drug-related problem in the emergency department in cocaine users (MARRIED-cocaine study). Clin Toxicol (Phila) 2012; 50:176182.

23. Burillo-Putze G, Borreguero león JM, García Dopico JA, Fernández Rodríguez JF, Pérez CarriLLO MA, Jorge PÉrez MJ et al. Incidence and impact of undisclosed cocaine use in emergency department chest pain and trauma patients. Int J Emerg Med 2008; 1: 169-172.

24. Domínguez Rodríguez A, Abreu González P, Enjuanes Grau C, Juárez Prera R, Arroyo Ucar E, Burillo-Putze G. El ligando soluble CD40 en pacientes con síndrome coronario agudo consumidores de cocaína. Emergencias 2011; 23:104-107.
25. Domínguez-Rodríguez A, Abreu-González P, Enjuanes-Grau C, Blanco-Palacios G, PÉrez-Carrillo MA, Burillo-Putze G. Oxidative stress and inflammatory markers in cocaine users with acute coronary syndrome. Med Clin (Barc) 2010; 134:152-155.

26. Burillo Putze G, Mesa Fumero J. Toxicología clínica, urgencias y urgencias pediátricas. Emergencias 2012; 24: 346-347.

27. Rosell Ortiz F, Mellado Vegel F, Fernández VaLle P, González Lobato I, Martínez Lara M, Ruiz Montero MM et al. Descripción y resultados iniciales del registro andaluz de parada cardiaca extrahospitalaria. Emergencias 2013; 25: 345-352.

28. Cone DC. Parada cardiaca extrahospitalaria en Andalucía: Un análisis inicial al registro de datos. Emergencias 2013; 25: 341-342.

29. Hernández García J, Medina Osuna A, Garzón SigLER R. Manejo extrahospitalario de los pacientes atendidos por dolor torácico en tres dispositivos móviles de cuidados críticos y urgencias. Emergencias 2013; 25: 13-22.

30. Fortuny E, NúÑEz Gil IJ, García-Rubira JC, Ruiz Mateos B, IbáÑez B, Gonzalo N et al. Relevancia clínica del tratamiento farmacológico completo precoz en el pronóstico del síndrome coronario agudo sin elevación del ST. Emergencias 2011; 23: 461-467.

31. Gómez-Hospital JA, Dallaglio PD, Sánchez-SAlado JC, Ariza A, Homs S, Lorente V et al. Impact on delay times and characteristics of patients undergoing primary percutaneous coronary intervention in the southern metropolitan area of Barcelona after implementation of the infarction code program. Rev Esp Cardiol 2012; 65: 911-918.

32. Llorens Soriano P, Martín-SÁnchez FJ, GonzÁlez Armengol JJ, Herrero Puente P, Jacob J, Bella Álvarez A et al. Perfil clínico del paciente con insuficiencia cardiaca aguda atendido en los servicios de urgencias: Datos preliminares del Estudio EAHFE (Epidemiology Acute Heart Failure Emergency). Emergencias 2008; 20: 154-163.

33. Aguirre Tejedo A, Miró O, Jacob Rodríguez A, Herrero Puente P, Martín-SÁnchez FJ, Alemany X et al. Papel del factor precipitante de un episodio de insuficiencia cardiaca aguda en relación al pronóstico a corto plazo del paciente: estudio PAPRICA. Emergencias 2012; 24: 438-446.

34. Jacob J, Llorens Soriano P, Martín-SÁnchez FJ, Herrero Puente P, Álvarez Argüelles A, PérezDuRÁ MJ et al. Valor pronóstico de la determinación urgente del péptido natriurético tipo $B$ en los servicios de urgencias en pacientes con insuficiencia cardiaca: estudio PICASU-1. Emergencias 2011; 23: 183-192. 
35. Miró O, Jacob J, Martín-SÁnchez FJ, Herrero Puente P, Pavón J, Pérez-Durá MJ et al. Implicaciones pronósticas de la posibilidad de determinar con carácter urgente el péptido natriurético tipo $B$ en el servicio de urgencias en pacientes con insuficiencia cardiaca aguda: estudio PICASU-2. Emergencias 2011; 23: 437-446.

36. Llorens Soriano P, Lau-Cheng A, Carratala JM, Climent E. Experiencia en el uso de tolvaptán en el servicio de urgencias en pacientes con insuficiencia cardiaca aguda e hiponatremia. Emergencias 2012; 24:126-129.

37. Llorens P, Miró O, Herrero P, Martín-Sánchez FJ, JACoB J, VAlero A ET al. Clinical effects and sa- fety of different strategies for administering intravenous diuretics in acutely decompensated heart failure: a randomised clinical trial. Emerg Med J 2014; (en prensa).

38. Llorens Soriano P, Miró O, Román F, Zapater P, Carbajosa Dalmau J, Llanos L. Eficacia de la administración precoz de levosimendán en urgencias en pacientes con insuficiencia cardiaca aguda: un ensayo clínico piloto aleatorizado. Emergencias 2012; 24: 268-276.

39. Martín-SÁnchez FJ, Fernández Pérez C. Llega la evidencia de los ensayos clínicos a EMERGENCIAS: el levosimendán en la insuficiencia cardiaca aguda Emergencias 2012; 24: 265267. 